\title{
Information retrieval curricula; contexts and perspectives
}

\author{
David Bawden \\ City University London, UK \\ db@soi.city.ac.uk \\ Jessica Bates \\ University College Dublin, Ireland \\ jess.bates@ucd.ie \\ Jela Steinerovu \\ Comenius University of Bratislava, Slovakia \\ jela.steinerova@fphil.uniba.sk \\ Pertti Vakkari \\ University of Tampere, Finland \\ pertti.vakkari@uta.fi \\ Polona Vilar \\ University of Ljubljana, Slovenia \\ polona.vilar@ff.uni-lj.si
}

\begin{abstract}
This paper reports work on curriculum development for information retrieval, in the wider context of information seeking and human information behaviour. It forms part of a larger EU-supported project, aimed at joint post-Bologna curriculum development for library and information science (LIS) in Europe.

No attempt is made to specify a single curriculum for information retrieval, and its associated topics. Instead an analysis of the wider area is made, in terms of its three aspects (human information behaviour, information seeking and information retrieval) and three perspectives or paradigms, by which it may be presented (human- or user-centred, culture-centred and system-centred). These led to a set of 28 topics, covering the whole theme, from which courses at a variety of levels (Batchelor, Master, or professional development), depth, and subject focus may be constructed.
\end{abstract}

Keywords: information retrieval; information seeking; human information behaviour; education and training; European curricula

\author{
1. INTRODUCTION \\ for in-service training and for continuing professional development (CPD). \\ 1. LIS curriculum in general \\ 2. Cultural heritage and digitisation of the cultural heritage \\ 3. Information literacy and learning \\ 4. Information seeking and information retrieval \\ 5. The information society \\ 6. Knowledge management \\ 7. Knowledge organisation \\ 8. The library in the multi-cultural information society \\ 9. Library and society in a historical perspective
}

The work described here forms part of a larger project - LIS Education in Europe: joint curriculum development and Bologna preparation project - supported by the European Union's SOCRATES/ERASMUS funding, and coordinated by EUCLID, the European Association for Library and Information Education and Research. This addressed the need for consideration of curricula in light of the Bologna process, which promotes harmonisation in higher education systems throughout Europe. Although the focus of the project is on curricula for higher education, some of the topics and themes dealt with - including this one - are equally applicable to design of shorter courses

The overall aim of the project is to deal with the whole of the library and information science (LIS) curriculum, and this has been done through twelve workgroups. The themes dealt with by these groups are: 
10. Mediation of culture in a European context

11. Practice and theory

12. Library management and promotion

This paper reports the findings of workgroup 4. Consolidated results for the whole project are available [1] and the work of this group is additionally reported elsewhere [2].

The group initially worked virtually, by email and a discussion forum, between January and July 2005, concluding the process face-to-face at a project meeting at the Danish Royal School of Librarianship and Information Science in August 2005. The project report was then prepared, and finalised in December 2005. Since then the group has continued its work, focusing on using the topics and perspectives to design specific curricula, and on seeking integration with other areas of the curriculum, specifically knowledge organisation.

\section{THE INFORMATION SEEKING AND RETRIEVAL TOPIC}

This topic is obviously large and extensive, covering the related but distinct subjects of information seeking (IS) and the more specific information retrieval (IR). Nonetheless, we decided that, in order to gain a coherent and comprehensive treatment, suitable as a basis for all courses - formal education, training and CPD - covering any aspect of this topic, it would have to be extended further, to include the still broader topic of human information behaviour (HIB). This is justifiable, not only because it is necessary in order to present a reasoned and logical curriculum, but also because of the increasing tendency to integrate information behaviour with the other two concepts, in research and scholarship

Broadening the topic in this way puts a strong constraint on the sort of analysis of the curriculum which can be carried out in practice. It is not possible to specify a single curriculum for any particular course covering such a wide area. Rather, we have sought to enumerate a list of topics from which such a course - whether it be for formal education, workplace training, or continuing professional development - may be constructed, and to analyse and present the paradigms, perspectives and relationships which may be applied in order to carry out the construction of a coherent and rational curriculum.

This is an area in which there is already a significant number of published models, frameworks and conceptual analyses[3,4]. We felt it important to use these whenever possible, rather than to create our own. We took the relationships between the three aspects of information seeking and retrieval (IS\&R) to be that illustrated in Wilson's nested model [5]. The widest outermost layer is human information behaviour. Within this, as a subset of information behaviour is information seeking. Within this, the innermost layer, is information retrieval, a specific form of information seeking.

Neither of the two inner layers can be understood without some appreciation of the layer outside them. This is the justification for introducing HIB into the IS\&R theme. It is also true that HIB itself cannot be fully understood without some appreciation of the wider contexts of human behaviour, but these are outside the scope of this paper.

\section{PERSPECTIVES ON INFORMATION SEEKING AND RETRIEVAL}

Three distinct perspectives may be used to analyse and understand IS\&R. They are not mutually exclusive, but rather give complementary insights. It is desirable that students (in all cases) and trainees (in some cases) are exposed to all three, although any particular course in the IS\&R theme may relate mainly to one of them. They are:

- human- or user-centred perspective

this focuses on the information needs and behaviour of individuals, emphasising studies of individual behaviour

- culture-centred perspective

this focuses on information seeking as an aspect of human culture, emphasising the ways in which information behaviour stems from, and contributes to, cultural groups, and is itself affected by social and organisational structures

- $\quad$ system-centred perspective this focuses on retrieval systems, emphasising system design and the evaluation of the effectiveness of system performance

In all of these perspectives, even when the focus may be on systems, the emphasis - distinguishing an LIS approach from an information technology (IT) or information systems approach should be on content, and on the 
information needs and use which require content, and on context, which determines how and why the systems are used, and which itself has several dimensions.

\section{CORE CONCEPTS OF INFORMATION SEEKING AND RETRIEVAL}

It is not our intention here to define and explain the core concepts of IS\&R; apart from space limitations, there are divergent views as to the best way of understanding these concepts, which it is not our task to debate (see Case [6], for a thoughtful discussion of several of these concepts). Rather, we list these concepts, simply as a means of delineating the theme. Any curriculum development based on the topics below will rely on a clear understanding of these concepts, and their relationships, together with an appreciation of the three perspectives outlined above.

These core concepts are:

- human information behaviour; information seeking; information retrieval

- knowledge; information; document; resource; retrieval system

- information need; information access; information use; becoming informed; information literacy

- relevance; utility; satisfaction; evaluation of information

- content (of information sources); context (of information use)

- knowledge organisation; indexing; vocabulary; information representation

\section{MODELS FOR INFORMATION SEEKING AND RETRIEVAL}

There are numerous relevant models representing aspects of the IS\&R theme. Use of such models will play a vital part in bringing coherence to the teaching of the complex area of IS\&R. The most appropriate models to use will depend on the topics being covered, and on the level and depth of the treatment. The models may be divided into four groups: broad frameworks for the understanding of IS\&R; conceptual models of information seeking; models of the searching process; and models of the retrieval mechanism (e.g. Boolean, best match, Bayesian). For examples and details of these models, see [1,2, 7, and 8]. It is desirable that students be introduced to examples of all four kinds; it may be sufficient for participants on short training courses to focus on a smaller number.

\section{TOPICS FOR INFORMATION SEEKING AND RETRIEVAL}

The group's attempt to divide the very broad theme of information seeking and retrieval into coherent and discrete chunks led to a set of 28 topics, intended to serve as a basis for discussing curriculum, content, teaching methods etc. The topics are these, with each topic noted as HIB, IS or IR, depending on which aspect of IS\&R it focuses on, or as Gen(eral) if it deals with all three.

$1 \quad$ Basic concepts and relationships

Gen Relationships between HIB, IS, IR

Three perspectives: human/user, culture, system

Concepts: information and knowledge; documents; typology of information resources

Relevant research methodologies; laboratory, operational, qualitative, quantitative

2 Overview of HIB

HIB Frameworks, concepts, models, theories

Research approaches and methods

Example topics

Historical development of studies

3 Overview of IS

IS Frameworks, concepts, models, theories

Research approaches and methods

Example topics

Historical development of studies 
$4 \quad$ Overview of IR

IR Frameworks, concepts, models, theories

Components of retrieval systems

Research approaches and methods

Example topics

Historical development of studies

$5 \quad$ Human information behaviour: people

HIB Individuals and groups

Occupation, age, activity, etc.

Characteristics: cognitive, social, cultural, organizational

$6 \quad$ Human information behaviour: sources and places

HIB Channels and media

Print, electronic, formal , informal, mass, local, ICTs

Places \& spaces - libraries, information centres, archives, museums, information grounds

$7 \quad$ Human information behaviour: patterns of behaviour

HIB Browsing, encountering, avoidance, anxiety, advantages of lack of

information, overload

Innovation and creativity

8 Information needs; nature and typology

HIB identifying information needs; users and non-users

$9 \quad$ Information literacy

HIB place of seeking/retrieval in wider context

teaching and supporting users to retrieve

10 Organising and using information

$H I B$

11 Role of information professionals

$H I B$

12 Information seeking in context

IS Occupational, professional, everyday life, etc.

13 Information seeking in specific domains (subjects)

IS relation to domain analysis

domain specific resources

14 Strategies and tactics for information seeking

IS Task-based and cognitive etc.

15 Relevance and satisfaction

IS concepts, typology, history, empirical studies

16 Person-centred information services

IS Developing services around needs, using research findings

17 Historical development of IR systems

IR associated IT: retrieval in different media - print, digital, network

18 Retrieval interfaces

IR $\mathrm{HCl}$, usability testing, personalisation.

Machine interfaces and interoperability, visualisation 
Typology of retrieval systems

$\mathrm{dbms}$, factual/numeric systems

bibliographic databases, full-text retrieval, e-journals, content management systems OPACS, digital library, managing digital resources Internet search engines, subject gateways, 'hidden web', semantic web Enterprise and knowledge management systems (Autonomy, Verity, Google, etc.)

\section{Specialised retrieval}

e.g. multimedia, images, audio, sounds, music, fiction, chemical structure, genome and protein sequence

Intelligent systems and techniques; cognitive aspects intelligent agents, $\mathrm{Al}$

data / text mining question-answering systems, recommender systems Cyc

\section{Retrieval tactics}

General and specific

Citation searching, bibliometrics. webliometrics

Retrieval language

Natural language processing, automatic indexing, classification, summarisation Multilingual systems, CLIR

\section{Metadata and controlled vocabularies}

Controlled vocabularies in retrieval ontologies, subject headings, thesauri, taxonomies, classifications, RDF, topic maps, concept retrieval / topic retrieval / latent semantic retrieval

Metadata and retrieval intellectual metadata creation: cataloguing, indexing, abstracting format and content standards

\section{Evaluation of systems and services}

IR system evaluation: TREC, metrics and other performance measures

User-oriented evaluation of information seeking and searching

\section{System design based on research findings}

New developments and future trends in HIB, IS and IR Current research topics

Evaluating research, evidence-based practice

The topics do not necessarily have equal weight, in the sense that some could generate more teaching material than others. Similarly, they could be used as the basis for courses at very different depth and level. All are suitable as a basis for courses at both Batchelor's and Master's level, and could also be used for in-service training, professional updating and CPD.

The topics may be used to form curricula at very different levels of granularity. At one extreme, an overview course for the whole IS\&R theme could be constructed by giving each topic one hour's presentation time, in a course of, for example, 10 sessions of 3 hours each. Conversely, if each topic were allowed 3 hours presentation, a course of the same duration would encompass 10 topics. This list of topics does not prescribe any extent of content. In a short and focused training course, a single topic could provide all the content, for, say, 6 hours presentation; while a single topic could also form the basis for a specialised elective course in higher education, with typically 30 contact hours. 


\section{ON-GOING WORK}

The conclusion of the LIS-EU project left several points in the IS\&R area requiring further analysis and reflection, including:

- use of the set of topics to construct some sample curricula in detail

- detailed exemplification of some of the differences in practice and perception in different parts of Europe

- a more detailed working out of the relations and links between IS\&R and KO, and other parts of the curriculum

- detailed analysis of how the three perspectives may be used to 'flavour' courses in IS\&R, without losing sight of generally applicable principles

These aspects are being pursued by some working group members in their own institutions as exemplars. It is hoped that further funding may be available in 2007 for a continuation of the LIS-EU work, in which the 'seeking and retrieval ' area would be integrated with knowledge organisation, dealing with what is arguably the core of the LIS discipline.

\section{REFERENCES}

[1] L Kajberg and L LDrring, European curriculum reflections on library and information science education, Royal School of Librarianship and Information Science,Copenhagen, 2005, available from http://www.db.dk/lis-eu.

[2] D Bawden, P Vilar, J Bates, I Cordeiro, J Steinerova, and P Vakkari, Europe-wide training programmes for information retrieval and information seeking, Proceedings ofOnline Information 2005, London: Learned Information Europe, pages 143-148.

[3] KE Pettigrew, R Fidel and $\mathrm{H}$ Bruce, Conceptual models in information behaviour research, Annual Reviews in Information Science and Technology, 2002, 55, 249-270.

[4] $\mathrm{K}$ J^rvelin and TD Wilson, On conceptual models for information seeking and retrieval research, Information Research, 2003, 9(1), paper 163, available from http://informationr.net/ir/9.1/paper163.html.

[5] TD Wilson Models in information behaviour research, Journal of Documentation, 1999, 55(3), 249-270.

[6] DO Case, Looking for information: a survey of research on information seeking, needs and behaviour, New York: Academic Press, 2002.

[7] P Ingwersen and $\mathrm{K}$ J^rvelin, The turn: integration of information seeking and retrieval in context, Dordrecht: Springer, 2005.

[8] K.E. Fisher, S Erdelez, and L McKechnie (eds.). Theories of information behaviour, Medford NJ: Information Today, 2005. 